\title{
Cancer Detection Rates in a Population-Based, Opportunistic Screening Model, New Delhi, India
}

\author{
Krithiga Shridhar', Subhojit Dey², Chandra Mohan Bhan³, Dipika Bumb ${ }^{3}$, \\ Jyostna Govil ${ }^{3}$, Preet K Dhillon ${ }^{1 *}$
}

\begin{abstract}
Background: In India, cancer accounts for 7.3\% of DALY's, 14.3\% of mortality with an age-standardized incident rate of 92.4/100,000 in men and 97.4/100,000 in women and yet there are no nationwide screening programs. Materials and Methods: We calculated age-standardized and age-truncated (30-69 years) detection rates for men and women who attended the Indian Cancer Society detection centre, New Delhi from 2011-12. All participants were registered with socio-demographic, medical, family and risk factors history questionnaires, administered clinical examinations to screen for breast, oral, gynecological and other cancers through a comprehensive physical examination and complete blood count. Patients with an abnormal clinical exam or blood result were referred to collaborating institutes for further investigations and follow-up. Results: A total of $n=3503$ were screened during $2011-12(47.8 \%$ men, $51.6 \%$ women and $0.6 \%$ children $<15$ years $)$ with a mean age of $47.8 \mathrm{yrs}( \pm \mathbf{1 5 . 1} \mathrm{yrs}) ; \mathbf{8 0 . 5} \%$ were aged $30-69$ years and $77.1 \%$ had at least a secondary education. Tobacco use was reported by $15.8 \%$, alcohol consumption by $11.9 \%$ and family history of cancer by $9.9 \%$ of participants. Follow-up of suspicious cases yielded 45 incident cancers (51.1\% in men, $48.9 \%$ in women), consisting of $55.5 \%$ head and neck (72.0\% oral), $28.9 \%$ breast, $6.7 \%$ gynecological and $8.9 \%$ other cancer sites. The age-standardized detection rate for all cancer sites was 340.8/100,000 men and 329.8/100,000 women. Conclusions: Cancer screening centres are an effective means of attracting high-risk persons in low-resource settings. Opportunistic screening is one feasible pathway to address the rising cancer burden in urban India through early detection.
\end{abstract}

Keywords: Opportunistic screening - India, cancer burden - age-standardized detection rate

Asian Pac J Cancer Prev, 16 (5), 1953-1958

\section{Introduction}

Over a million new cancer cases were diagnosed in India in 2012 (Ferlay et al., 2013) and the number is predicted to rise by almost one-third to $1,320,928$ in 2020 (NCRP, 2013). The estimated age-standardized rate (ASR) for all cancer sites was 94.0/100,000 for incidence and 64.5/100,000 for mortality in 2012 (Ferlay $\mathrm{J}$ et al., 2013). In India, 1 in 20-24 persons are estimated to die of cancer in his/her lifetime (Dikshit et al., 2012). The three most common cancer sites in Indian men are lung $(\mathrm{ASR}=11.0 / 100,000)$, oral $(\mathrm{ASR}=10.1 / 100,000)$ and stomach $(\mathrm{ASR}=8.6 / 100,000)$ and in women are breast $(\mathrm{ASR}=25.8 / 100,000)$, cervix $(\mathrm{ASR}=22.0 / 100,000)$ and colo-rectum (ASR=5.1/100,000) (Ferlay J et al., 2013) (NCRP 2013). These sites, except for cervix in women, continue to show increasing or stable trends of incidence across a majority of population-based cancer registries (PBCRs) in India (NCRP, 2013) (Rath, 2014). Of all PBCRs, the New Delhi cancer registry recorded the maximum number of new cancers $(29,027)$ in 2008-09 and the most common cancer sites were breast, cervix, oral and lung (NCRP, 2013).

There are no nationwide screening programmes for cancer in India (Chalkidou et al., 2014). Cancerrelated health delivery services are also less developed (Sankaranarayanan et al., 2010) (Nair et al., 2013) with large variations in the accessibility of diagnostic and treatment facilities across regions and between urban and rural populations (Sankaranarayanan et al., 2010; Dikshit et al., 2012) (Pal and Mittal, 2004). In spite of effective, low-cost techniques of early detection for common cancer sites such as oral, breast and cervix (Kumar et al., 2011b), the majority are detected in advanced stages (Pal and Mittal 2004; Sankaranarayanan et al., 2010; Dikshit et al., 2012; Thomas et al., 2012; Nair et al., 2013). This is due to low awareness of the disease, its etiology or signs/ symptoms, social stigma of cancer, and limited access to screening, early diagnosis and health services (Kumar et al., 2011b; Raj et al., 2012; Thomas et al., 2012; Pati et al., 2013). As a result, fewer than $30 \%$ of patients in India survive more than 5 years after diagnosis (Mallath 
et al., 2014). Expensive cancer treatment (an average of INR 41,311 for treatment and medicines even in a public tertiary care setting) (Nair et al., 2013) can lead to catastrophic health spending, whereby households are pushed into poverty due to healthcare spending (Mahal et al., 2013; Nair et al., 2013).

Given the rising burden of cancer, which is expected to increase by $81 \%$ in low-and middle income countries (LMICs) in 2030 (Adams and Doherty, 2014) and escalating treatment costs, there is an urgent need to support and implement cost-effective early detection programmes. While organized population-based mass screening programmes for common cancer sites such as breast, cervix and oral (mouth and tongue) are still in trials in India (Sankaranarayanan et al., 2007; 2011a; 2011b; 2013; Mittra et al., 2010), or being evaluated in certain state-wide models (e.g., Tamil Nadu) (Krishnan et al., 2013) an opportunistic screening model for cancers remains an alternate strategy for cancer control (Pal and Mittal 2004; Bhatla et al., 2008; Lee et al., 2010; Mauad et al., 2011; Kulkarni et al., 2013). Here, we report agetruncated (30-69 years) and age-standardized cancer detection rates from an opportunistic cancer screening centre model over a 2-year period in New Delhi, India.

\section{Materials and Methods}

The Indian Cancer Society (ICS), a 63-yr old non-governmental organization (NGO) conducts screening camps, awareness workshops and hosts a walk-in, population-based, screening centre for oral, oropharyngeal, prostate, breast and gynecological cancers. All screening examinations (described in detail below) are performed and reviewed by an ear, nose and throat (ENT) specialist, general surgeon and a female gynecologist.

The general public receives information about the ICS cancer screening centre through radio talks, awareness camps/workshops/seminars, brochures that are distributed at fund-raisers, shopping malls, camp locations and also during awareness talks at colleges and schools, audiovisual aids and ICS newsletters. Fund-raising events, social media networking, collaborations with local NGO's and a gender resource centre facilitate a wider outreach across various sections of the community. Radio talks are broadcast to the National Capital Region (New DelhiNational Capital Region such as Gurgaon, Faridabad, Noida) carried out bi-monthly every 1 st and 3rd Friday for 20-30 minutes on topics regarding risk factors for male and female cancers, prevention, treatment and palliative care availabilities by ICS volunteers. Awareness talks are conducted 5-6 times per month at schools, colleges, corporate organizations, residential communities and urban slums by ICS volunteers, survivors and other members.

Adult men and women (aged 15+ years) and also children (aged $<15$ years) who visit the screening centre, are registered with an interviewer-administered, structured questionnaire on socio-demographic characteristics (education, occupation), medical history, family history of cancer, diabetes and tuberculosis, reproductive history, tobacco use and diet (such as intake of eggs, fresh fish, dry fish, meat, pickle etc.). All men and women (children only if required) undergo a series of examinations and screening investigations that include a clinical breast exam, gynecological examination and pap smear for women, oral visual examination, ENT examination and a chest $\mathrm{X}$-ray done using digital X-ray machine (Siemens model no.5205, Hitachi). Height, weight and blood pressure are taken and a random blood sample is collected for a complete blood count (CBC) analysis using automatic blood analyzer (Epson LX-300-II) manufactured by Swelab alfa company-Sweden for all patients. Patients with an abnormal clinical exam, blood result or papsmear are advised for further investigations such as a human papilloma virus DNA test, mammography, ultrasound, DEXA scan, biopsy and followed up with referring oncologists at local cancer hospitals, such as All India Institute of Medical Sciences (AIIMS), Army R\&R hospital, Rajiv Gandhi cancer institute and others). Patients with suspicious findings are tracked by phone to assist newly diagnosed patients navigate their care pathway. The average time between presenting for screening to time of diagnosis at ICS is 15-20 days.

Age-truncated detection rates were calculated for men and women aged 30-69 years who were screened at ICS between 2011 and 2012 using total number of newly detected cases (for each cancer site) during that period divided by the total screened population, among those aged 30-69 years. The results are presented for each year. For the New Delhi PBCR, we estimated age-truncated incidence rates per 100,000 for the year 2012, using linear extrapolation from New Delhi PBCR incidence rate data in 2008-2009 and from the 2001 and 2011 census data

Table 1. Socio-demographic and Life-style Characteristics of the Screening Population (2011-12)

\begin{tabular}{|c|c|c|c|}
\hline Characteristics & $\begin{array}{c}\text { Men } \\
\mathrm{N}=1676 \\
(47.8 \%)\end{array}$ & $\begin{array}{l}\text { Women } \\
\mathrm{N}=1811 \\
(51.6 \%)\end{array}$ & $\begin{array}{c}\text { Total } \\
\mathrm{N}=3503^{*}\end{array}$ \\
\hline Age & $\begin{array}{l}50.2(15.8) \\
(15-97 \mathrm{yrs})\end{array}$ & $\begin{array}{l}45.7 \text { (13.6) } \\
(16-89 \text { yrs) }\end{array}$ & $\begin{array}{c}47.8(15.1) \\
(9-97 \mathrm{yrs})\end{array}$ \\
\hline \multicolumn{4}{|l|}{ Education } \\
\hline Primary/secondary & 28.3 & 31.4 & 30 \\
\hline Graduate & 36.1 & 38.1 & 37.2 \\
\hline Professional & 10.5 & 3.5 & 6.8 \\
\hline Missing & 25 & 26.8 & 25.9 \\
\hline \multicolumn{4}{|l|}{ Family history } \\
\hline Yes & 10.2 & 9.6 & 9.9 \\
\hline No & 69.7 & 72.7 & 71.2 \\
\hline Missing & 20.1 & 17.8 & 18.9 \\
\hline \multicolumn{4}{|l|}{ Tobacco } \\
\hline Never & 58.2 & 82.6 & 70.9 \\
\hline Current & 29.2 & 3.4 & 15.8 \\
\hline Missing & 12.5 & 13.9 & 13.3 \\
\hline \multicolumn{4}{|l|}{ Alcohol } \\
\hline Never & 63.1 & 84.4 & 74.2 \\
\hline Current & 23.3 & 1.3 & 11.9 \\
\hline Missing & 13.5 & 14.2 & 13.9 \\
\hline \multicolumn{4}{|l|}{ Diet } \\
\hline Vegetarian & 32.7 & 42.7 & 38.0 \\
\hline Non-vegetarian & 50.7 & 45.1 & 47.7 \\
\hline Missing & 16.5 & 12.2 & 14.3 \\
\hline Newly detected cancers & s 23 & 22 & 45 \\
\hline
\end{tabular}


for New Delhi. Age-standardized detection rates were calculated using the Segi world standard (1968).

\section{Results}

A total of $n=3503$ men, women and children visited the ICS detection centre for screening during 2011-12 ( $47.8 \%$ men, $51.6 \%$ women and $0.6 \%$ children $<15$ years) with a mean age of $47.8 \mathrm{yrs}( \pm 15.1 \mathrm{yrs})$. The majority of participants, $80.5 \%$, were aged $30-69$ years, more than three-quarters were literate and $77.1 \%$ had at least a primary/secondary education. Tobacco use was reported by $15.8 \%$ (29.2\% in men, $3.4 \%$ in women), alcohol consumption by $11.9 \%$ (23.3\% in men, $1.3 \%$ in women) and family history of cancer by $9.9 \%$ (10.2\% in men, $9.6 \%$ in women) (Table 1). Newly diagnosed cancers over the 2-year study period ( $\mathrm{n}=45$; all in adult men and women) were representative of the screening population with respect to gender (51.1\% in men, $48.9 \%$ in women), use of tobacco (29.2\% in men, $3.4 \%$ in women) and alcohol (23.3\% in men, $1.3 \%$ in women). The site distribution consisted of $55.5 \%$ head and neck (72.0\% oral (C01-06), $28.0 \%$ tonsil, pharynx and larynx (C09-14, C32), 28.9\% breast (C50), 6.7\% gynecological (C53-54) and 8.9\% other cancer sites such as gall bladder, urinary bladder and leukemia (C23, C67, C42 respectively) (Table 2, Figure 1). The most commonly detected three cancer sites were oral (955/100,000 men and 110/100,000 women),

Table 2. Distribution of Newly Detected Cancer Sites in ICS Screening Population (2011-12)

\begin{tabular}{lcccc}
\hline & $\begin{array}{c}\text { Oral } \\
(\mathrm{C} 00-06)^{*}\end{array}$ & $\begin{array}{c}\text { Breast } \\
(\mathrm{C} 50)^{*}\end{array}$ & $\begin{array}{c}\text { Gynecological } \\
(\mathrm{C} 53-54)^{*}\end{array}$ & $\begin{array}{c}\text { Head and Neck } \\
(\mathrm{C} 09-\mathrm{C} 14, \mathrm{C} 32)^{*}\end{array}$ \\
\hline Total cases & 18 & 13 & 3 & 7 \\
$(\mathrm{C} 23, \mathrm{C} 67, \mathrm{C} 42)^{*}$
\end{tabular}

*C00- lip C01-Base of tongue C02- Other parts of tongue C03-Gum C04-Floor of mouth C05-Palate C06- Other parts of mouth; C53-Cervix Uteri; C54- Corpus Uteri; C09-Tonsil C10-Oropharynx C11-Nasopharynx C12-Pyriform sinus C13-Hypopharynx C14Other ill-defined sites of lip, oral cavity and pharynx; C32- Larynx; C23- Gall bladder; C67- Urinary bladder; C42- Hematopoietic system

Table 3. Comparison of Age-standardized Detection Rates of cancer in the ICS Screening Population (2011-12) and Estimated Age-standardized Incidence Rates of New Delhi Cancer Registry (2012) and India (GloboCan 2012)

\begin{tabular}{lcccccc}
\hline ASR/100,000 & \multicolumn{3}{c}{ Women } & \multicolumn{2}{c}{ Men } \\
\cline { 2 - 7 } For all ages & $\begin{array}{c}\text { ICS } \\
\text { ASDR }\end{array}$ & $\begin{array}{c}\text { New Delhi ASR ** } \\
\text { ASR }^{\dagger}\end{array}$ & $\begin{array}{c}\text { India } \\
\text { ASDR }\end{array}$ & $\begin{array}{c}\text { ICS } \\
\text { ASR }^{\dagger}\end{array}$ & New Delhi ASR** & India \\
\hline Oral cancer & 26.4 & 5.8 & 4.3 & 239.4 & 16.9 & 10.1 \\
$\begin{array}{l}\text { Breast cancer } \\
\text { Gynecological cancer } \\
\text { (corpus uteri and cervix) }\end{array}$ & 195.3 & 22 & 25.8 & - & - & - \\
All sites & 47.4 & 7.5 & & - & - & - \\
\hline
\end{tabular}

*All sites (for ICS) in men include urinary bladder $(\mathrm{n}=1)$; larynx $(\mathrm{n}=2)$; leukemia $(\mathrm{n}=1)$; tonsil $(\mathrm{n}=2)$ and neck $(\mathrm{n}=1)$ and all sites in women include gall bladder $(n=1)$; urinary bladder $(n=1)$ and neck $(n=1) * *$ New Delhi estimated ASR (incidence rate) for the year 2012 based on $2001-2011$ census population, New Delhi PBCR data in 2008-2009, and linear extrapolation of changing incidence rates and census population $†$ GloboCan estimated ASR (incidence rate) for 2012; ASDR - Age-standardized detection rates; ASR-Age-standardized incidence rates

Table 4. Total Number of Cases and Comparison of age-truncated Detection Rates between the ICS Screening Population (2011-12) and Estimated Age-Truncated Incidence Rates of New Delhi Cancer Registry (2012).

\begin{tabular}{lcc}
\hline & \multicolumn{2}{c}{ Number of cases over the 2-year period } \\
ICS & Men (n=1306) \\
\cline { 2 - 3 } Total detected cases (30-69years) & Women $(\mathrm{n}=1514)$ & 21 \\
All sites* & 17 & 15 \\
Oral cancer & 2 & - \\
Breast cancer & 10 & - \\
Gynecological cancers (corpus uteri \& cervix) & 2 & -
\end{tabular}

\begin{tabular}{lcccc}
\hline \multicolumn{4}{c}{ Age truncated (30-69 years) detection rates (per 100,000) } \\
\hline & ICS & New Delhi $^{\dagger}$ & ICS & New Delhi $^{\dagger}$ \\
\hline All sites & $561 *$ & 141.5 & $804 *$ & 349.8 \\
Oral cancer & 66 & 10.6 & 374.5 & - \\
Breast cancer & 330.5 & 47.1 & - \\
Gynecological cancers (corpus uteri \& cervix) & 61 & 15.1 & - \\
\hline
\end{tabular}

* All sites in men for ICS (30-69 years) include urinary bladder $(n=1)$ larynx $(n=2)$ leukemia $(n=1)$ tonsil $(n=2)$ and neck $(n=1)$ and all sites in women (30-69 years) include gall bladder $(n=1)$ urinary bladder $(n=1)$ and neck $(n=1) \dagger$ Estimated age truncated incidence rate for the year 2012 based on 2001-2011 census population, New Delhi PBCR data in 2008-2009, and linear extrapolation of changing incidence rates and census population; ATDR- Age-truncated detection rates; ATR-Age-truncated incidence rates 


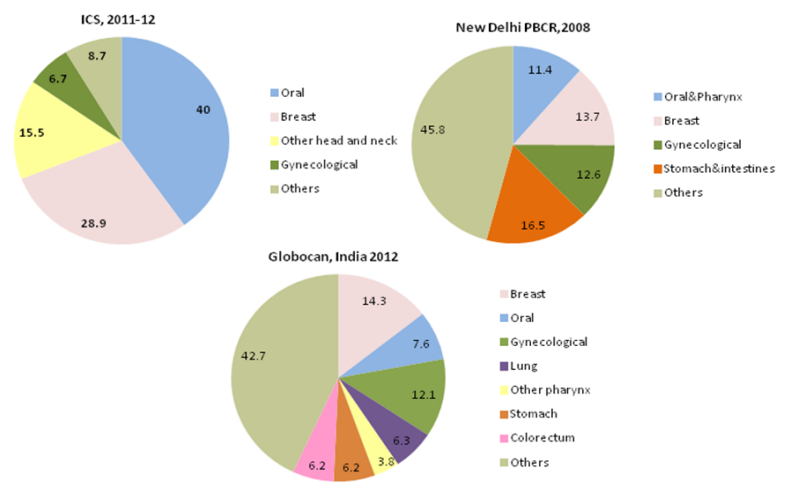

Figure 1. Comparison of Cancer Distribution at ICS (2011-12) with New Delhi PBCR (2008) and Indian Globocan (2012)

breast (718/100,000 women) and gynecological cancers $(166 / 100,000$ women) defined as corpus uteri (C54) and cervix (C53) combined (data not shown). The age standardized detection rate (ASDR) at ICS for all cancer sites in men was 340.8/100,000 and 329.8/100,000 in women per year (Table 3). The age-truncated (30-69 yrs) detection rate (ATDR) of all cancer sites was 804/100,000 in men and 561/100,000 in women per year. The agetruncated (30-69 yrs) detection rate (ATDR) for specific sites were 574.5/100,000 in men and 66/100,000 in women for oral; 330.5/100,000 in women for breast and $61 / 100,000$ in women for gynecological cancers per year (Table 4).

\section{Discussion}

We observed very high cancer detection rates over a 2-year period of a population-based opportunistic cancer screening model in New Delhi. Over 3500 men and women visited the centre for opportunistic screening, yielding age-truncated (30-69 years) detection rates of $804 / 100,000$ in men and 561/100,000 in women per year, which were 2 times and 4 times higher for men and women respectively, when compared to the estimated age truncated incidence rates of the New Delhi PBCR for the year 2012. The age-standardized detection rates for all cancer sites and all ages were $340.8 / 100,000$ and $329.8 / 100,000$ in women per year, which are 2-5 times higher than both the New Delhi PBCR and GLOBOCAN estimated age-standardized incidence rates for 2012. These rates actually indicate that men are more likely to come forward for screening with their symptoms than women. Oral cancer in men and breast cancer in women are the leading sites of cancer detection at ICS, New Delhi.

To conduct systematically organized, populationbased, mass screening programmes nationally or regionally, is still a matter of debate as the most effective model for cancer control in resource-constrained societies (Palencia et al., 2010). In the absence of nation- or statewide systematic, organized, population-level screening programmes with adequate referral and diagnostic pathways and follow-up (Lee et al., 2010) (Kulkarni et al., 2013) (Madlensky et al., 2003), opportunistic, lowcost, population-based screening with a referral system to affordable tertiary cancer centres, offers a model with access to primary and secondary prevention of cancer (Palencia et al., 2010) (Lee et al., 2010) (Madlensky et al., 2003).

Even in developed countries where organized screening models have been in place for decades, obstacles such as low participation rates among young adults and lower SES groups, difficulties in completing investigative procedures and late diagnosis of disease are still reported (Arbyn et al., 2009) (Lee et al., 2010). On the contrary, in regions where organized programmes are not practiced, opportunistic screening yielded better turnover rates (Palencia et al., 2010) and the downward stage shift of the disease was also comparable to organized programmes (Bordoni et al., 2009) consistently due to general awareness and more responsible medical systems (Palencia et al., 2010) (Bordoni et al., 2009). In developing countries such as India, organized mass screening programmes for common cancer have shown mixed results for oral cancer (Brocklehurst et al., 2010) and are still in the trial phase for breast and cervix (Mittra et al., 2010) (Krishnan et al., 2013). Yet these initiatives have led to considerable improvement in the availability of cancer screening facilities and awareness among the public (Sankaranarayanan et al., 2011b). Given the complexities and barriers in scaling up screening programs, particularly with health systems and human resource constraints for referral and follow-up (Mallath et al., 2014) (Samiei 2014), a population-level, opportunistic cancer screening model offers a practical alternative in the current scenario. One of the limitations of our present report is the missing information on socio-demographic and life-style risk factor data which accounted for about $15-25 \%$ for different variables. However, the opportunistic screeners in this study did not differ from the general population in terms of tobacco use, alcohol consumption, family history of cancer and prevalence of vegetarianism (Bhawna 2013) (Arnold et al., 2009) when compared to national level data on these risk factors. Another limitation is incomplete information on stage of diagnosis to evaluate down-staging in this screened population.

Aside from opportunistic screening programmes at the population level, hospitals conduct similar programmes through oral cancer screening (Thomas et al., 2012), cervical cancer screening in women utilizing health care services for other reasons (Kulkarni et al., 2013) or in single-visit 'see and treat' approach (visual inspection with acetic acid and loop excision) (Singla et al., 2012) as well as training of paramedics (Bhatla et al., 2004) (Bhargava et al., 1993). The major focus of such programs till date has been on cervical cancer (Bhargava et al., 1993; Nene et al., 1996; Bhatla et al., 2004; Bhatla et al., 2009; Singla et al., 2012) and with current trends in India, there is a need to expand these services to both genders and to other commonly occurring cancers, and to account for geographic variations in the cancer burden (NCRP, 2013).

The mean age of opportunistic screeners in New Delhi was similar to the age distribution reported in the literature, (Thomas et al., 2012) (Kumar et al., 2011a; Kumar et al., 2011b) (Kulkarni et al., 2013) (Bhatla et al., 2004) with the majority of participants aged $30-65$ years, although 
participants were more educated and of higher SES, which is consistent with reports from other opportunistic screening programs (Lee et al., 2010) (Palencia et al., 2010) (Arbyn et al., 2009). In India, opportunistic screening camps - both population- and hospital-based successfully recruit people from lower SES and education backgrounds (Kumar et al., 2011a) (Kumar et al., 2011b) (Bumb et al., 2014) (Bhatla et al., 2004), although the referral and treatment pathways for these populations are often constrained by obstacles (Sankaranarayanan et al., 2010). ICS conducts mobile screening camps twice a week in low SES communities and screens over 10,000 people annually, however there are limited data on follow-up so we could not assess detection rates from camps.

Early detection methods in a screened-naïve population will detect pre-clinical prevalent disease (Morrison 1992), and screening centres/programmes are more likely to attract high-risk or symptomatic populations leading to higher detection rates; rates as high as 190.0-288.9 cases/10,000 screened women for cervical cancer have been reported earlier (Singla et al., 2012; Kulkarni et al., 2013) (Bhatla et al., 2009). As a result, the detection rates are not comparable to incidence rates reported by PBCR's, but they provide a high catchment rate with adequate referral and diagnostic pathways, in a population under-served by health promotion and screening. Interestingly, they yielded higher rates in men than women, although true incidence rates reveal the reverse from registries (NCRP, 2013) and nationwide estimates (Ferlay et al., 2013), suggesting gender and socio-economic differences in awareness and acknowledgment of symptoms and risk factors. The follow-up of suspicious cases and early diagnosis at the population level in an opportunistic screening model is a challenge (Lee et al., 2010) (Madlensky et al., 2003) which can be addressed through collaborations and strong linkages between cancer screening and early detection centres and affordable cancer treatment facilities.

In conclusion, opportunistic cancer screening offers a relevant, important strategy for early detection of cancer in a low-resource country with rising rates of cancer and its NCD risk factors. Increased awareness of such screening programmes, which are heavily subsidized and very low cost, can further increase the impact and benefit of such programs to all members of a low-resource society. Barriers for self-referral in persons of lower education and socio-economic status need to be understood and addressed for higher levels of awareness and participation in India's cancer early detection and control programs.

\section{References}

Adams C and Doherty RM (2014). Advocacy for Cancer control. World Cancer Report. B.W.S and C.P. W, Available at www. iarc.fr> Publications〉 Books> World Cancer Report, 2014.

Arbyn M, Rebolj M, De Kok IM, et al (2009). The challenges of organising cervical screening programmes in the 15 old member states of the European Union. Eur J Cancer, 45, 2671-8.

Arnold F, Parasuraman S, Arokiasamy P, et al (2009). Nutrition in India National Family Health Survey. I. NFHS 3, 2005 06 Mumbai: International Institute for Population Sciences. Calverton, Maryland, USA: ICF Macro.
Bhargava VL, Verma K, Sharma R, et al. (1993). A hospitalbased study on the use of paramedical personnel for clinical downstaging of cancer cervix. Indian J Med Res, 98, 65-8.

Bhatla N, Dar L, Rajkumar Patro A, et al (2008). Human papillomavirus-type distribution in women with and without cervical neoplasia in north India. Int J Gynecol Pathol, 27, 426-30.

Bhatla N, Gulati A, Mathur SR, et al (2009). Evaluation of cervical screening in rural North India. Int J Gynaecol Obstet, 105, 145-9.

Bhatla N, Mukhopadhyay A, Joshi S, et al (2004). Visual inspection for cervical cancer screening: evaluation by doctor versus paramedical worker. Indian J Cancer, 41, 32-6.

Bhawna G (2013). Burden of smoked and smokeless tobacco consumption in India - results from the global adult tobacco survey India (GATS-India)- 2009-201. Asian Pac J Cancer Prev, 14, 3323-9.

Bordoni A, Probst-Hensch NM, Mazzucchelli L, et al (2009). Assessment of breast cancer opportunistic screening by clinical-pathological indicators: a population-based study. Br J Cancer, 101, 1925-31.

Brocklehurst P, Kujan O, Glenny AM, et al (2010). Screening programmes for the early detection and prevention of oral cancer. Cochrane Database Syst Rev, 11, 4150.

Bumb D, Shridhar K, Dhillon PK, et al (2014). Oral visual examination for early detection of potentially malignant mucosal disorders in an opportunistic population. Oral Oncol, 50, 3-4.

Chalkidou K, Marquez P, Dhillon PK, et al (2014). Evidenceinformed frameworks for cost-effective cancer care and prevention in low, middle, and high-income countries. Lancet Oncol, 15, 119-31.

Dikshit R, Gupta PC, Ramasundarahettige C, et al (2012). Cancer mortality in India: a nationally representative survey. Lancet, 379, 1807-16.

Ferlay J, Soerjomataram I, Ervik M, et al (2013). GLOBOCAN 2012 v1 .0 Cancer Incidence and Mortality Worldwide: IARC CancerBase No. 11 [Internet]. Lyon, France: International Agency for Research on Cancer; 2012 Available from: http:// globocan.iarc.fr

Krishnan S, Madsen E, Porterfield D, et al (2013). Advancing cervical cancer prevention in India: implementation science priorities. Oncologist, 18, 13-25.

Kulkarni PR, Rani H, Vimalambike MG, et al (2013). Opportunistic screening for cervical cancer in a tertiary hospital in Karnataka, India. Asian Pac J Cancer Prev, 14, 5101-5.

Kumar Y, Mishra G, Gupta S, et al (2011a). Cancer screening for women living in urban slums--acceptance and satisfaction. Asian Pac J Cancer Prev, 12, 1681-5.

Kumar YS, Mishra G, Gupta S, et al (2011b). Level of cancer awareness among women of low socioeconomic status in Mumbai slums. Asian Pac J Cancer Prev, 12, 1295-8.

Lee HY, Park EC, Jun JK, et al (2010). Trends in socioeconomic disparities in organized and opportunistic gastric cancer screening in Korea (2005-2009). Cancer Epidemiol Biomarkers Prev, 19, 1919-26.

Madlensky L, Goel V, Polzer J, et al (2003). Assessing the evidence for organised cancer screening programmes. Eur J Cancer, 39, 1648-53.

Mahal A, Karan A, Fan VY, et al (2013). The economic burden of cancers on Indian households. PLoS One, 8, 71853.

Mallath MK, Taylor DG, Badwe RA, et al (2014). The growing burden of cancer in India: epidemiology and social context. Lancet Oncol, 15, 205-12.

Mauad EC, Silva TB, Latorre MR, et al (2011). Opportunistic screening for skin cancer using a mobile unit in Brazil. $B M C$ 
Dermatol, 11, 12.

Mittra I, Mishra GA, Singh S, et al (2010). A cluster randomized, controlled trial of breast and cervix cancer screening in Mumbai, India: methodology and interim results after three rounds of screening. Int J Cancer, 126, 976-84.

Morrison AS (1992). Screening in Chronic Disease. Oxford University Press, 3-20.

Nair KS, Raj S, Tiwari VK, et al (2013). Cost of treatment for cancer: experiences of patients in public hospitals in India. Asian Pac J Cancer Prev, 14, 5049-54.

NCRP National Cancer Registry Program: Bangalore. (2013). Three year report of Population Based Cancer Registries, 2009-2011, Indian Council Med Res.

Nene BM, Deshpande S, Jayant K, et al. (1996). Early detection of cervical cancer by visual inspection: a population-based study in rural India. Int J Cancer, 68, 770-3.

Pal SK and Mittal B (2004). Improving cancer care in India: prospects and challenges. Asian Pac J Cancer Prev, 5, 226-8.

Palencia L, Espelt A, Rodriguez-Sanz M, et al (2010). Socioeconomic inequalities in breast and cervical cancer screening practices in Europe: influence of the type of screening program. Int J Epidemiol, 39, 757-65.

Pati S, Hussain MA, Chauhan AS, et al (2013). Patient navigation pathway and barriers to treatment seeking in cancer in India: a qualitative inquiry. Cancer Epidemiol, 37, 973-8.

Raj S, Piang LK, Nair KS, et al (2012). Awareness regarding risk factors, symptoms and treatment facilities for cancer in selected states of India. Asian Pac J Cancer Prev, 13, 4057-62.

Rath GK (2014). Cancer control in India:Cancer care through a four tier system. World Cancer Report B. W. S and C. P. $\mathrm{W}$, vailable at www.iarc.fr > Publications $>$ Books > World Cancer Report 2014

Samiei M (2014). Health system strengthening for cancer control. World Cancer Report. B. W. S and C. P.W, available at www. iarc.fr > Publications > Books > World Cancer Report 2014

Sankaranarayanan R, Esmy PO, Rajkumar R, et al (2007). Effect of visual screening on cervical cancer incidence and mortality in Tamil Nadu, India: a cluster-randomised trial. Lancet, 370, 398-406.

Sankaranarayanan R, Ramadas K, Thara S, et al (2011a). Clinical breast examination: preliminary results from a cluster randomized controlled trial in India. J Natl Cancer Inst, 103, 1476-80.

Sankaranarayanan R, Ramadas K, Thara S, et al (2013). Long term effect of visual screening on oral cancer incidence and mortality in a randomized trial in Kerala, India. Oral Oncol, 49, 314-21.

Sankaranarayanan R, Sauvaget C, Ramadas K, et al (2011b). Clinical trials of cancer screening in the developing world and their impact on cancer healthcare. Ann Oncol, 22, 20-28.

Sankaranarayanan R, Swaminathan R, Brenner H, et al (2010). Cancer survival in Africa, Asia, and central America: a population-based study. Lancet Oncol, 11, 165-73.

Singla S, Mathur S, Kriplani A, et al (2012). Single visit approach for management of cervical intraepithelial neoplasia by visual inspection \& loop electrosurgical excision procedure. Indian J Med Res, 135, 614-20.

Thomas V, Rema Devi S, Jeyaseelan V, et al (2012). Mucosal disorders with oral epithelial dysplasia risk--development of a simple screening tool for general health care setting. Oral Oncol, 48, 671-7. 